\title{
Gripping an object based on inspection of slip events for a robotic hand model
}

\begin{abstract}
An effective grasping operation is required in intelligent robotic hands to address the wellknown challenge that occurs during the gripping process. One of the most important issues is detecting and addressing the slip situation; otherwise, stable grasps will not be achieved properly. This study investigated robotic re-gripping operations based on slip event indication. Tactile pressure sensors are utilized to discover the physical interaction between robotic claws and a particular object during the gripping operation. Slip signal detection is executed by implementing a rotary encoder device that was provided in a robotic hand model. The robotic system has attempted to accomplish the re-gripping mission autonomously. Therefore, an automatic feedback control algorithm is developed to perform re-gripping tasks based on the distance at which an object has slipped. Experimental findings present the correlation between the required forces for an object to re-grip securely and the distance at which an object has slipped. This approach was demonstrated as Hooke's law.
\end{abstract}

Keyword: Re-gripping operation; Robotic hand model; Slip detection device; Tactile pressure sensors 